PRINT ISSN 1119-8362

Electronic ISSN 1119-8362
Full-text Available Online at https://www.ajol.info/index.php/jasem

http://ww.bioline.org.br/ja
J. Appl. Sci. Environ. Manage.

Vol. 24 (12) 2131-2138 December 2020

\title{
Sacharomyces Cerevisiae Tolerance to Hyper-Osmolarity in Aerobic Glucose-Limited Chemostat Culture
}

\author{
1,2 AGBO, SO; ${ }^{3}$ NEBO, UC; ${ }^{2}$ OGAUGWU, CE; ${ }^{1}$ VAN TUIJL, A \\ ${ }^{I}$ Department of Molecular Cell Physiology, Vrije University Amsterdam, De Boelelaan 1105, 1081 HV Amsterdam, The Netherlands. \\ ${ }^{2}$ Department of Animal and Environmental Biology, Federal University Oye-Ekiti, Ekiti State, Nigeria. \\ ${ }^{3}$ Department of Microbiology, Federal University Oye-Ekiti, Ekiti State, Nigeria. \\ *Corresponding Author Email: sozoemena@hotmail.com; stanley.agbo@fuoye.edu.ng
}

\begin{abstract}
Different mechanisms contribute to regulate cellular functions in order to cope with threats from physiological stress conditions. As a fundamental response to balance excess water loss and restore turgor, Saccharomyces cerevisiae subjected to increased osmolarity accelerate intracellular glycerol biosynthesis and accumulation as a compatible solute. This study assessed cellular response to sorbitol-induced osmolarity in aerobic glucose-limited chemostat culture at various levels of the glycerol flux. Cell number declined slightly without any substantial increase in dry weight and total protein contents following exposure to $1 \mathrm{M}$ sorbitol that lasted for $90 \mathrm{~min}$. On the other hand, total glycerol levels increased over time in different yeast cultivations corresponding with enhanced glycerol-3-phosphate dehydrogenase 1 (GPD1) transcript levels, which led to a steady and intensified protein product. These results support literature evidence of accumulated intracellular glycerol regulation at different levels and further increase curiosity to understand yeast tolerance in various applications including bakery, brewery and wine making or leavening of bread under different osmotic conditions.
\end{abstract}

DOI: https://dx.doi.org/10.4314/jasem.v24i12.19

Copyright: Copyright (C) 2020 Agbo et al. This is an open access article distributed under the Creative Commons Attribution License (CCL), which permits unrestricted use, distribution, and reproduction in any medium, provided the original work is properly cited.

Dates: Received: 05 October 2020; Revised: 25 November 2020; Accepted: 15 December 2020

Keywords: Saccharomyces cerevisiae, glycerol, sorbitol, osmotic stress, chemostat

Cellular response to ecosystem perturbations at sublethal strength can be studied using molecular markers as well as whole cell events. Though widespread in nature mostly at tolerable levels, environmental stress in the form of habitat alteration implies large-scale climatic shifts due to heat, cold, chemical pollution, hypoxia, free radicals, nutrient starvation, salinity or hyper-osmolarity, outside optimal physiological range that may induce adverse effects in biota (David et al., 2017; Abhishek and Ramakrishnan, 2016). Some previous studies utilized visible perceptible endpoints including motility, defective development, morphology or weight as effect indicators thereby making it possible for subtle yet effective stress levels to evade detection and ultimately lead to risk underestimation (Forbes, 1999; Abhishek and Ramakrishnan, 2016). Other studies addressed different aspects of cellular stress response in relation to signal transduction (Hotamisligil and Davis, 2016; Toone and Jones, 1998), gene activation and protein induction (Albertyn et al., 1994), metabolite flux and transport (Neves et al., 2004). However, research information obtained from such studies did not clearly integrate findings to link cellspecific responses and adaptation to environmental stress. Due to a need to establish interconnectedness of stressors at various regulatory levels, researchers developed an integrative model of yeast response to osmotic shock (Klipp et al., 2005). The model presents a representative approach that requires time course experimental data on cellular adaptive responses. More so, it integrates hierarchical regulation of cellular stress thus making it necessary to establish links between transcript levels, cellular proteins and metabolite profiles. It is a crucial relationship considering that mRNA molecules that may be induced by stressors are not functional entities. Similarly, abundance of proteins which are functional cellular entities after adequate post-translational modifications does not necessarily correlate with increased cellular activity and therefore may require complementary metabolic studies (Rossell et al., 2005). In actual fact, a vertical genomics approach is required to more clearly understand regulations of inducible effects at multiple regulatory levels especially at subtly low stress levels.

For instance, in a typical industrial process, yeast cells are exposed to increased osmolarity in high salt and sugar levels, leading to imbalance in cellular water and turgor (Reijenga, 2002). Apart from shrinkage in size, consequences of water loss may lead to increased 
concentrations of intracellular metabolites, thereby leading to alteration in architectural integrity of cytoskeleton. The MAP kinase pathway is activated as a compensatory response to maintain internal homeostasis (De Nobel et al., 2000; Mager and Siderius, 2002). Other regulatory mechanism to counter increased osmolarity includes an enhanced biosynthesis of inert compatible solutes and subsequent shut down of facilitator channels to accumulate intracellular metabolites. As an essential compatible solute, yeast accumulates intracellular glycerol in response to decreased extracellular water activity but also to facilitate cellular redox balance by replenishing cellular NAD (Nevoigt and Stahl, 1997; Bakker et al., 2001; Klipp et al., 2005). In brief, glycerol is produced from dihydroxyacetone phosphate (DHAP) in a two-step process that requires reduction of DHAP by glycerol-3-phosphate dehydrogenase (GPD) followed by dephosphorylation of the resulting intermediate by glycerol-3phosphatase (GPP) (Siderius et al., 2000; Remize et al., 2001). Other studies suggest that yeast shut down glycerol transport channels and accelerate uptake from surrounding medium (Luyten et al., 1995; Tamás et al., 1999). However, it is possible that efflux of cellular water and enhanced glycerol uptake from surrounding medium happen concurrently since cells appear to use this mechanism to counteract excessive outflow. In the end, this mechanism can help to maintain cellular volume, turgor pressure and normal biological activities.

Considering this background, yeast applications in baking, brewery and wine making or leavening of bread expose them to elevated temperature, varying substrate and product concentrations, aeration, $\mathrm{pH}$, high salt and sugar concentrations (Cereghino and Cregg, 2000; Ostergaard et al., 2000). While high salt and sugar levels affect the physiology of yeast, signal transduction pathways may be activated with consequences on activity of relevant enzymes (Hotamisligil and Davis, 2016). The objective of this study is to assess different levels of intracellular regulation and adaptation of $S$. cerevisiae to sorbitolinduced osmotic stress.

\section{MATERIALS AND METHODS}

Cell culture conditions: S.cerevisiae CEN.PK 113.7d was obtained from the Department of Molecular Cell Physiology, Vrije University Amsterdam. An aliquot of cells was taken from $-80^{\circ} \mathrm{C}$, thawed and grown in a defined CBS-C medium as batch cultivation. The culture was shaken overnight in a water bath $\left(30^{\circ} \mathrm{C}\right)$ at 200rpm. Chemostat cultivation was applied to obtain larger volumes in controlled culture conditions for optimal growth and yield. Moreover, it is an ideal setup with better controlled experimental conditions with respect to cell density, aeration, substrate and product concentrations, and temperature. Also, accumulation of metabolic products that may inhibit cellular growth is minimized while supply of fresh medium is maintained. The chemostat setup consists of a water bath and $\mathrm{pH}$ electrode to regulate temperature and $\mathrm{pH}$, respectively. A motor-driven stirrer ensured constant mixing to achieve homogeneity of culture. Based on study design, the medium vessels were carefully connected in such a way that one fed the culture through an inlet, while the other collected out-flowing culture as waste. A level sensor ensured that balance of volume is maintained while restraining the accumulation of products and buildup of cell density in time. The $1 \mathrm{~L}$ capacity chemostat fermenter was inoculated with a $100 \mathrm{ml}$ of culture and maintained under constant stirring (800rpm) at $30^{\circ} \mathrm{C}$ for $24 \mathrm{hr}$. Medium was allowed into the fermenter at a dilution rate of $0.1 \mathrm{~h}^{-1}$, while culture $\mathrm{pH}$ was maintained at 5.0 with $0.5 \mathrm{M}$ Potassium hydroxide. Adequate sterile aeration was supplied at a speed of $500 \mathrm{ml} / \mathrm{min}$ using a millipore filter device. Samples were taken for use in determining dry weight and optical density (OD). In addition to HPLCassisted determination of ethanol yield and consumption, off-gas measurements were conducted to assess consumption of oxygen and release of carbon dioxide. The chemostat culture attained steady state at $40 \mathrm{hr}$ and subsequently, samples were taken for cell count, dry weight, glycerol determination, transcript levels, whole cell protein and western blotting.

At steady state, however, a known culture volume was removed and replaced by an equal volume of sorbitol to yield a final concentration of $1 \mathrm{M}$. Afterward, the culture medium reservoir was replaced by another reservoir containing sorbitol and glucose. Cell samples were taken every $10 \mathrm{~min}$ during a $90 \mathrm{~min}$ period of osmotic shock. Similarly, we conducted corresponding sorbitol-induced shock and sampling of cells at $0,2,4,6,8,10,15,30$ and $60 \mathrm{~min}$.

Coulter counter and dry weight measurements: Samples were taken from the culture at intervals and placed on ice. Subsequently, the samples were diluted with isotone prior to cell counting on a Bechman Coulter Multisizer II. To determine dry weight, predried and pre-weighed nitrocellulose filter of $0.4 \mu \mathrm{m}$ pore size was mounted on filter installation and equilibrated with demineralized water. Shortly, a culture of $1 \mathrm{ml}$ was filtered under vacuum (Wertheim, Germany). To rid cells of salt, three repeated wash steps were performed each with $1 \mathrm{ml}$ of water before filters were placed in glass Petri dishes and oven-dried 
for $20 \mathrm{hr}$ at $60^{\circ} \mathrm{C}$. Afterward, dry weights of samples were determined.

Determination of glycerol levels: Glycerol levels were determined enzymatically according to Albertyn et al. (1994) but with minor modifications. The glycerol assay was performed in well plates on NOVOSTAR (BMG, LABTECH) at an absorbance of $340 \mathrm{~nm}$. For total glycerol, $0.5 \mathrm{ml}$ culture was quenched in $55 \mu 1$ of $35 \%$ perchloric acid. Extracellular glycerol concentration was determined from supernatant that resulted from centrifuging $1 \mathrm{ml}$ of culture. Intracellular glycerol was determined as the difference between total and external glycerol concentrations.

Quantitative Polymerase Chain Reaction ( $q P C R)$ : Cell samples were taken from culture at intervals and snap-frozen in liquid nitrogen prior to storage at $-80^{\circ} \mathrm{C}$ until use. Later, samples were thawed on ice followed by RNA isolation in hot phenol according to Köhrer and Domde (1991) but with minor modification. Specific primers were used to amplify corresponding genes by qPCR in order to determine relative transcript abundance (Table 1).

Table 1. Sequences of primers used to amplify target genes

\begin{tabular}{llll}
\hline DESCRIPTION & GENBANK & FORWARD & REVERSE \\
\hline HTA1 (225W) & YDR225W & AACGTTACCATTGCCCAAGGT & CCTTGGTAGCCTTGGCAGACT \\
PDI1 (043C) & YCL043C & GCCATCCACGACATGACTGA & TCTTGTCGCTCAATTCGTCAA \\
YGPD1 & YDL022W & TGAAGAACGTTGTTGCTTAGG & ACTCTTGGGTGGCAGCAGAAG \\
YGPD2 & YOL059W & TCCGCTGGTGTTGAGATC & ACCGGTCTTGGCCATGTATG \\
YGPP1 & YIL053W & CGAAGTTCAGGTGCTGA & TGTCACGGGTACCAGAGGTG \\
YGPP2 & YER062C & ATGGTTCGAGCATCTGGGAAT & TCTGGATGAGGCTTACCCTGTT \\
YHOG1 & YLR113W & CGCTGATCTGCCTGTCGATAC & CTGTCCATCACTGCCACCAA \\
YSTL1 & YDR536W & GAGATTCCCCGTGTCAATGC & CAACGTGGCGATTCAGGTAGT \\
\hline
\end{tabular}

Preparation of cell extracts: Cells were harvested by centrifuging samples taken at intervals during osmotic shock and washing them in ice cold sonication buffer (100mM K $\mathrm{HPO}_{4}, 10 \mathrm{mM} \mathrm{KH_{2 }} \mathrm{PO}_{4}, 0.75 \mathrm{~g}$ EDTA). Later, cells were taken up in sonication buffer with end concentration of $1 \mathrm{mM}$ DTT and a cocktail of phosphatase inhibitor. Cell-free extracts were obtained by mechanical disruption of cell wall following sonication with acid washed glass beads $(250-500$ microns) according to a previous protocol and stored at $-20^{\circ} \mathrm{C}$ until use (Norbeck et al., 1996). Protein concentrations were determined by bicinchoninic acid (BCA) method using bovine serum albumin (BSA) as standard.

Western blotting: Pellet of cells harvested from $5 \mathrm{ml}$ of culture was resuspended in $1 \mathrm{ml}$ of ice-cold demineralized water. Crude cell extracts for Western blot analysis were prepared by breaking up cells with glass beads in a fast prep homogenizer (Bio 101, Thermo Savant Fastprep FP120, Cedex, France). Cell debris was removed by centrifugation while protein contents were determined by BCA method. Mobility and separation of proteins were facilitated by sodium dodecyl sulfate-polyacrylamide (SDS) gel $(12 \%)$ electrophoresis at a constant voltage $(\sim 100 \mathrm{~V})$. An overnight blotting on PVDF membrane was performed at $20 \mathrm{~V}$ and $50 \mathrm{~mA}$. The membrane was blocked with $5 \%$ nonfat dried milk in $1 \%$ TBST buffer containing $0.05 \%$ Tween 20 for about $2 \mathrm{hr}$ at room temperature. Four successive wash steps in $1 \%$ TBST were performed prior to $1 \mathrm{hr}$ incubation with a primary antibody ( $\alpha$-GPD1). A commercially available primary antibody was raised against GPDH and yeast hexokinase (HXK). In this context, hexokinase served as control to determine non-variable induction in yeast when subjected to osmotic stress but also to ensure that protein samples loaded were equal. Later, a secondary antibody (donkey anti goat IgG HRP-linked) in blocking solution was applied on a washed membrane and incubated for $1 \mathrm{hr}$. Detection was performed with a Lumi-Light Kit (Merck KGaA, Darmstadt, Germany) while luminescence was measured on a BIO RAD Quantity One.

\section{RESULTS AND DISCUSSION}

Cell count: Osmotic stress regulation can be studied by considering variations of intracellular molecules but also at whole cell level.

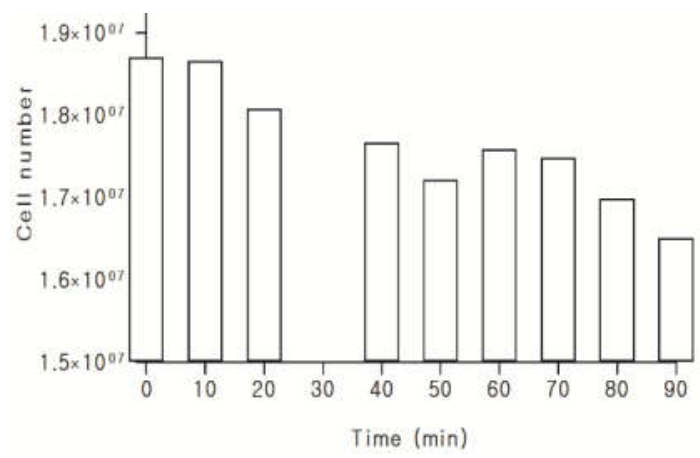

Fig 1. Cell counts during osmotic stress in $1 \mathrm{M}$ sorbitol

It is expected that yeast cells subjected to osmotic shock would devout substantial energy to restore homeostasis while normal cell division is slowed down. In this context, cellular stress due to sorbitol exposure appeared to have only slightly affected the 
formation and release of buds to contribute to the overall number of cells. As a result, we observed only a slight decrease in cell number after 90 min of shock (Figure 1). An aliquot of cell culture diluted by an equal volume of sorbitol appeared to account for the slight decrease in cell number between steady state and 0 min of osmotic shock.

Dry weight and whole cell protein: It is important to assess cellular response by determining whether stress-induced storage carbohydrates and proteins accumulate over time due to osmotic stress. We report here that over the period of osmotic shock, cells did not show any substantial increase in dry weight or protein at 60 and 90 min of cultivation (Figure 2). This observation is consistent with our previous unpublished study that rather showed a decline in cellular trehalose during osmotic stress.

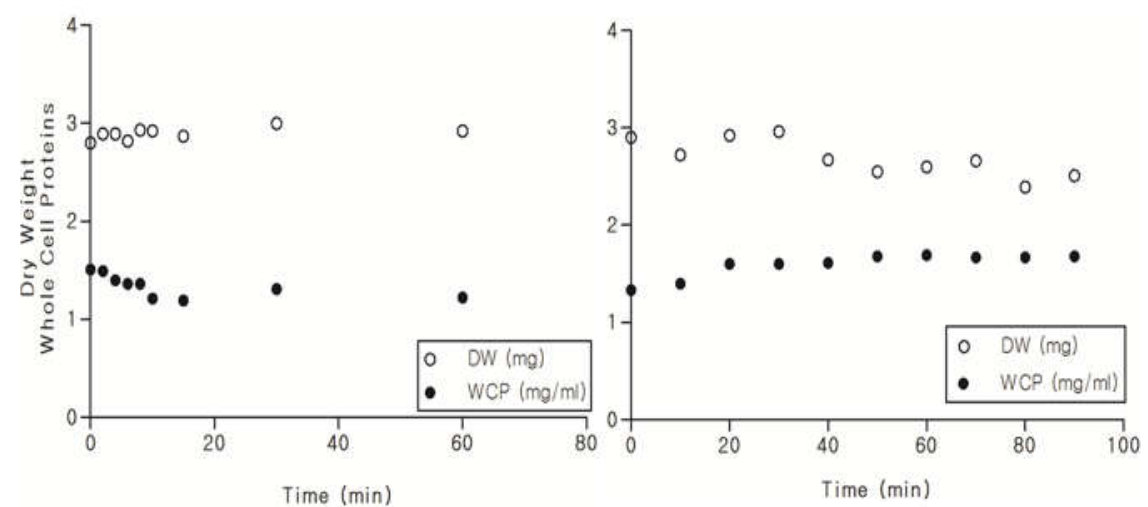

Fig 2. Cellular dry weight and whole cell protein levels at 60 and 90 min of $1 \mathrm{M}$ sorbitol shock

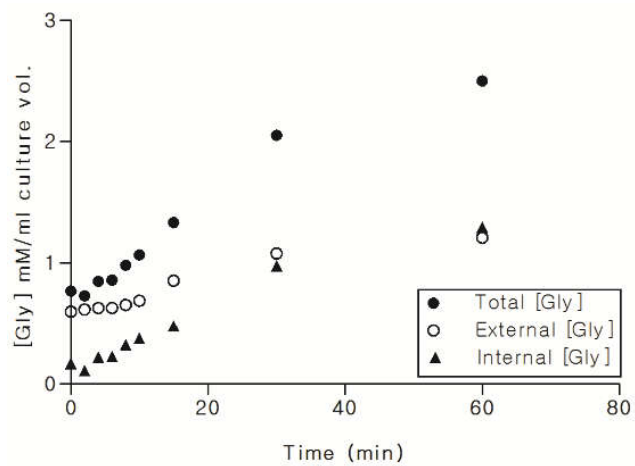

Fig 3. Intracellular, extracellular and total glycerol levels in yeast cells subjected to $1 \mathrm{M}$ sorbitol

Cellular glycerol: Considering the role of glycerol in regulation of osmotic stress, we attempted to quantify it using an HPLC. However, it was not successful, probably because of elevated sorbitol level in samples, which interfered with the stability of the chromatograph. However, we noted a time dependent increase in total glycerol level following osmotic stress in different cell cultivations (Figure 3). Internal glycerol levels appeared to be more rapid when compared to estimated extracellular glycerol (Figure 3).

Western Blotting: Following yeast exposure to osmotic stress, we assessed GPD1 induction by monitoring intensities of bands over time. There was hardly any enhanced induction of GPD1 at steady state. However, induction increased steadily and intensified over time for the duration of osmotic stress (Figure 4). This observation was similar in both randomized and non-randomized samples, though more evident as sampling became more frequent (Figure 4). Hexokinase was used as control to ensure that volume of samples loaded were equal and confirms that it does not vary in cells subjected to osmotic stress. Further step to quantify the intensity of bands revealed apparent increase in GPD1 (Figure 4).

qPCR Assay: Transcript level for osmo-sensitive gene, STL1, utilized as control revealed a rapid increase until about $30 \mathrm{~min}$ but decreased considerably for the remaining duration of osmotic shock (Figure 5). Similarly, GPP2 showed a relatively high expression both in rapidity and fold change (Figure 5). In contrast, HOG1 and GLO1 were hardly induced following 
exposure to osmotic stress. Other genes GPD1 and GPP1 that are crucial isogenes in glycerol biosynthesis pathway were similarly regulated (Figure 5). However, when one compares the observed differential regulation patterns to counterpart isogenes
GPD2 and GPP2, it is possible that the latter genes play different regulatory roles in $S$. cerevisiae adaptation to osmotic stress and maintenance of redox balance (Bakker et al., 2001).

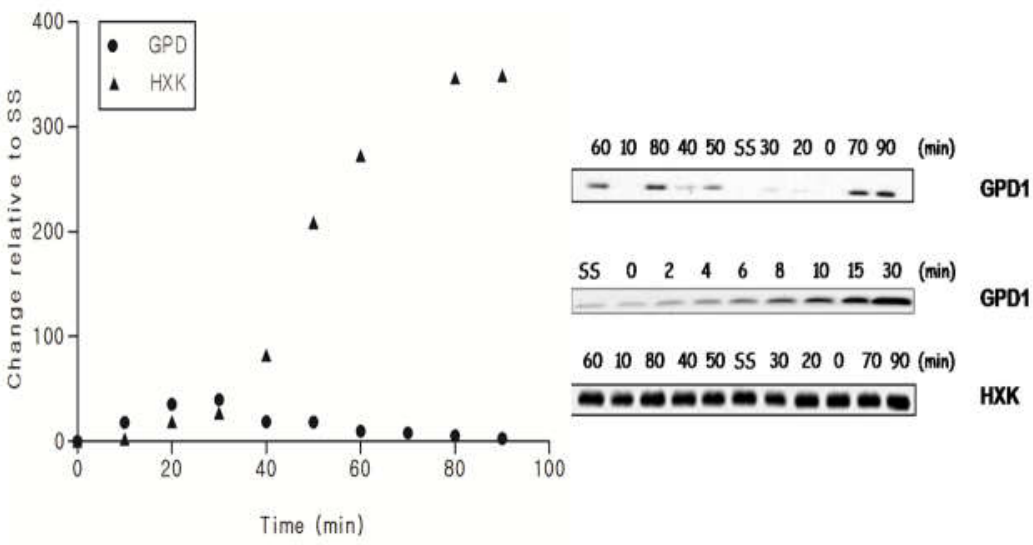

Fig 4. Band intensity corresponding to band patterns in a Western blot analysis to assess GPD induction relative to HXK control in a 1M sorbitol shock
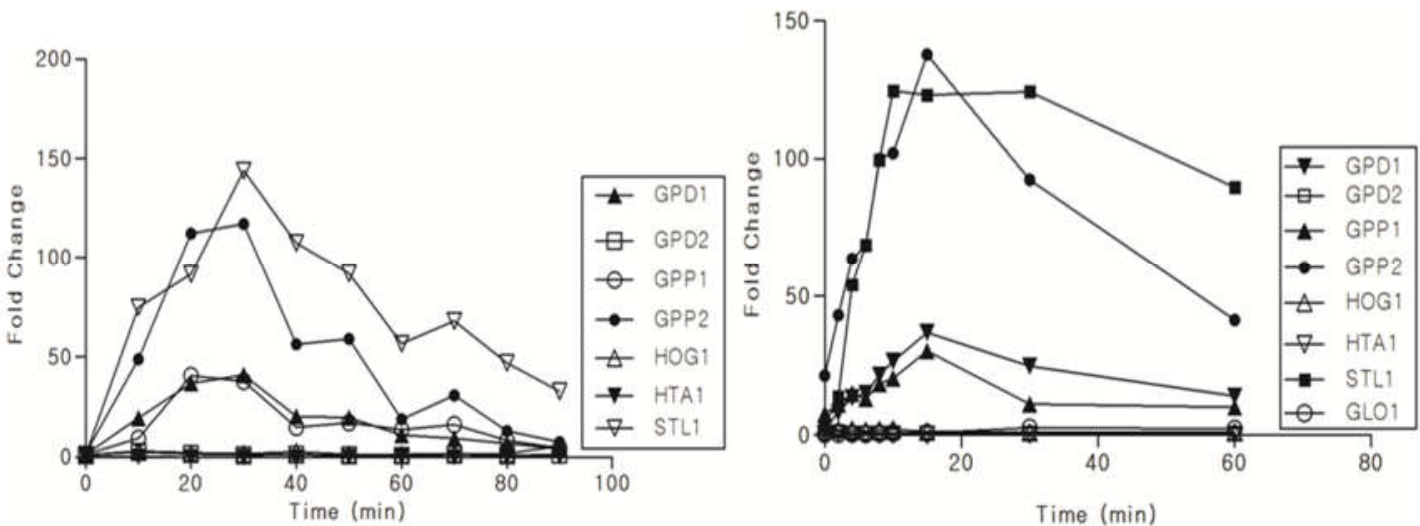

Fig 5. Transcriptional changes in yeast cells subjected to osmotic stress and normalized to weakly osmo-sensitive gene, PDI1, at 60 and 90 min cultivation time

Results obtained from this study suggest that normal internal osmotic balance was perturbed in yeast cells subjected to increased osmolarity. Hence, yeast cells mobilized various mechanisms required to restore homeostasis, which were evident in cellular abundance, dry weight and osmolyte biosynthesis. Based on this, it is necessary to assess cellular osmotic stress regulations at various levels in well-defined steady state chemostat conditions. We noted, however, that cells did not reveal any substantial increase in dry weight and cell number due to osmotic stress. Rather, biosynthesis and accumulation of glycerol were enhanced. This is in agreement with a general view that osmolytes enable yeast to counterbalance gradient to restore turgor. These observations suggest that even after normalization to cellular volume, concentrations were still sufficiently high to compensate for extracellular turgor. Therefore, when one considers that glycerol biosynthesis and accumulation constitute a critical aspect of cellular adaptive response to hyperosmolarity, it is natural to expect that cells would recruit various rapid homeostatic mechanisms to counter osmotic imbalance and cellular water loss. It ensures that cells do not suffer dehydration by promoting swift synthesis and accumulation of compatible osmolytes until balance in osmotic gradient is restored. Even previously, elevated level of intracellular glycerol had been associated with altered membrane permeability to glycerol while under osmotic stress (Capusoni et al., 2019; Albertyn et al., 1994). This view is in agreement with our preliminary unpublished result that revealed increased glycerol yield and accumulation within the first $1.5 \mathrm{hr}$ of a $1 \mathrm{M}$ sorbitol shock. Based on this, it is important to 
investigate if a corresponding osmolyte will take over role of balance in osmotic gradient under prolonged osmotic stress condition. Considering that yeast cells are routinely faced with osmotic tension, one would assume that a threshold cytosolic glycerol accumulative capacity is required to restore cellular turgor and maintain homeostasis. This view holds only if severity of osmotic stress remains within a tolerable sublethal range. However, other studies reported that trehalose confers protection on cells under osmotic stress to guard them from dessication (Wiemken, 1990; Werner-Washburne et al., 1993). What is not clear is whether metabolite accumulation can directly elicit gene expression. Reports from batch culture studies have shown that transcript levels can vary in certain members of HSP70 multigene family as cells enter different growth phases (Werner-Washburne et al., 1989; Choder, 1991). Even so, it is not certain whether a similar decline in transcript level would occur as cells approach stationary phase in chemostat culture. While there is no clear evidence to support the above statement, the discontinuity of correlation between cellular glycerol yield and transcript abundance after about 30 min of shock is believed not to have solely resulted from switch to alternative carbon source. Even though glucose was in limited supply, this study provided adequate carbon source throughout the duration of osmotic shock. It is recommended that further analysis be performed to establish correlations between duration of shock, level of intracellular glycerol accumulation and translation of osmo-responsive GPD1. Given that glycerol synthesis is a multi-step process that involves conversion of substrates into several intermediate products and metabolites, it is possible for cells to accelerate or slow down this process when in high or low demand for intracellular glycerol, respectively. While in high demand, other relevant enzymes facilitate glycerol synthesis to meet the needs of cells. In particular, glycerol-3-phosphate dehydrogenase (GPD) catalyzes the first reaction from dihydroxyacetone-phosphate to glycerol-3-phosphate, which is then converted to glycerol by glycerol-3phosphatase (GPP) (Albertyn et al., 1994; Norbeck et al., 1996). In a successful assay, however, we did not observe any measurable activity of GPD. Activity of this enzyme may have been inhibited by a high level of phosphate in samples. The speculative phenomenon of high phosphate interference with enzyme activity is suspected to have occurred in GPP activity assay. Nonetheless, it is known that glycerol synthesis and accumulation may reach a threshold beyond which there is no further increase. Since there is enormous cytosolic release of inorganic phosphate due to reduction of glycerol-3-phosphate, it is likely that even trace amount of phosphate is enough to inhibit activity of the enzyme. What is not clear is whether cells adopt a buildup in phosphate to regulate enzyme activity. Even if the above assumption is true, one may wonder why no measurable enzyme activity was detected before a threshold phosphate level was attained. The fact is that cellular transition from optimal growth conditions to sudden hyper-osmolarity is expected to stimulate response at the level of protein. A mutation study of GPD1 confirmed its adaptive role in osmotic stress transition, while mutant osmo-sensitivity occurred as a consequence of inability to accumulate considerable level of glycerol (Albertyn et al., 1994). It is evident that gene product of GPD1 is required to ameliorate sudden loss of cellular water. Even more specifically in this study, we demonstrated that GPD induction is crucial for osmo-tolerance. The relative ease of GPD responsiveness upon shock, even within a short time frame, appears to corroborate its biomarker candidacy among other osmo-inducible proteins. Likewise, it is striking that glycerol flux appeared to reach its maximum before GPD induction became visible. Nonetheless, GPD has consistently shown tremendous ease of detection. While this consistency is evident from this study, it further confirms that GPD band intensities (relative to hexokinase as control) increased with duration of osmotic stress. This observation corresponds well with band patterns even in randomized samples.

Conclusion: We report that glycerol is a necessary inert compatible solute, massively induced and accumulated intracellularly for osmo-tolerance. Induction of GPD1 in yeast increased correspondingly with exposure time and this is in agreement with transcript abundance. These results provide evidence that yeast cells can regulate osmotic stress at different transcriptional and metabolic levels.

\section{REFERENCES}

Abhishek, S; Ramakrishnan, S (2016). Osmoregulation in Saccharomyces cerevisiae via mechanisms other than the high-osmolarity glycerol pathway. Microbio.162:1511-1526.

Albertyn, J; Hohmann, S; Thevelein, JM; Prior, BA (1994). GPD1 which encodes glycerol-3phosphate dehydrogenase, is essential for growth under osmotic stress in Saccharomyces cerevisiae, and its expression is regulated by the high-osmolarity glycerol response pathway. Mol. Cell Biol. 14:4135-4144.

Bakker, BM; Overkamp, KM; van Maris, JA; Kötter, P; Luttik, AH; van Dijken, JP; Pronk, JT (2001). Stoichiometry and compartmentation of NADH 
metabolism in Saccharomyces cerevisiae. FEMS Microbiol. Rev. 25:15-37.

Capusoni, C; Arioli, S; Donzella, S; Guidi, B; Serra, I; Compagno, C (2019). Hyper-osmotic stress elicits membrane depolarization and decreased permeability in halotolerant marine Debaryomyces hansenii strains and in Saccharomyces cerevisiae. Front. Microbiol. doi: https://doi.org/10.3389/fmicb.2019.00064.

Cereghino, JL; Cregg, JM (2000). Heterologous protein expression in the methylotrophic yeast Pichiapastoris. FEMS Microbiol. Rev. 24:45-66.

Choder, M (1991). A general topoisomerase 1dependent transcriptional repression in the stationary phase of yeast. Genes Dev. 5:23152326.

David, CH; Takeshi, F; Ruth, G; Zhihua, H; Rumen, I; Giorgio, P; Song, L (2017). Multilevel regulation of abiotic stress responses in plants. Front Plant Sci. 8:1564.

De Nobel, H; Ruiz, C; Martin, H; Morris, W; Brul, S; Molina, M; Klis, FM (2000). Cell wall perturbation in yeast results in dual phosphorylation of the Slt2/Mpk1 MAP kinase and in an Slt2-mediated increase in FKS2-lacZ expression, glucanase resistance and thermotolerance.

MICROBIOLOGY 146:2121-2132.

Forbes, VE (1999). Studying stress in ecological systems: implications for ecological risk assessment and risk management. Ecol. Appl. 9:429-430.

Hotamisligil, GS; Davis, RJ (2016). Cell signaling and stress responses. Cold Spring Harb Perspect Biol. 8(10): a006072.

doi: 10.1101/cshperspect.a006072.

Klipp, E; Nordlander, B; Krüger, R; Gennemark, P; Hohmann, S (2005). Integrative model of response yeast to osmotic shock. Nat. Biotechnol. 23:975-982.

Kohrer, K; Domdey, H (1991). Preparation of high molecular weight RNA. Method Enzymol. 194:398-405.

Luyten, K; Albertyn, J; Skibbe, F; Prior, BA; Ramos, J; Thevelein, JM; Hohmann, S (1995). Fps1, a yeast member of the MIP-family of channel proteins, is a facilitator for glycerol uptake and efflux and it is inactive under osmotic stress. EMBO J. 14:1360- 1371 .

Mager, WH; Siderius, M (2002). Novel insights into the osmotic response of yeast. FEMS Yeast Res. 2:251-257.

Neves, L; Oliveira, R; Lucas, C (2004). Yeast orthologues associated with glycerol transport and metabolism. FEMS Yeast Res. 5:51-62.

Nevoigt, E; Stahl, U (1997). Osmoregulation and glycerol metabolism in the yeast Saccharomyces cerevisiae. FEMS Microbiol. Rev. 21:231-241.

Norbeck, J; Pahlman, AK; Akhtar, N; Blomberg, A; Adler, L (1996). Purification and characterization of two isoenzymes of DL-Glycerol-3-phosphate from Saccharomyces cerevisiae. J. Biol. Chem. 271:13875-13881.

Ostergaard, S; Olsson, L; Nielsen, J (2000). Metabolic engineering of Saccharomyces cerevisiae. Microbiol. Mol. Biol. R. 64:34-50.

Reijenga, K (2002). Dynamic control of yeast glycolysis. PhD Thesis.Vrije University Amsterdam, The Netherlands.

Remize, F; Barnavon, L; Dequin, S (2001). Glycerol export and glycerol-3-phosphate dehydrogenase, but not glycerol phosphate, are rate limiting for glycerol production in Saccharomyces cerevisiae. Metab. Eng. 3:301-312.

Rossell, S; van der Weijden, CC; Kruckeberg, AL; Bakker, BM; Westerhoff, HV (2005). Hierarchical and metabolic regulation of glucose influx in starved Saccharomyces cerevisiae. FEMS Yeast Res. 5:611- 619.

Siderius, M; van Wuytswinkel, O; Reijenga, KA; Kelders, M; Mager, WH (2000). The control of intracellular glycerol in Saccharomyces cerevisiae influences osmotic stress response and resistance to increased temperature. Mol. Microbiol. 36:1381- 1390.

Tamás, MJ; Luyten, K; Sutherland, FCW; Hernandez, A; Albertyn, J; Valadi, H; Li, H; Prior, BA; Killian, SG; Ramos, J; Gustafsson, L; Thevelein, JM; Hohmann, S (1999). Fps1p controls the accumulation and release of the compatible solute glycerol in yeast osmoregulation. Mol. Microbiol. 31:1087-1104. 
Toone, WM; Jones, N (1998). Stress-activated signaling pathways in yeast. Genes Cells 3:485498.

Werner-Washburne, M; Berker, J; Kosic-Smithers, J; Craig, EA (1989). Yeast Hsp70 RNA levels vary in response to the physiological status of the cell. J. Bacteriol.171:2680-2688.
Werner-Washburne, M; Braun, E; Johnston, GC; Singer, RA (1993). Stationary phase in the yeast Saccharomyces cerevisiae. Microbiol. Rev.57:383-401.

Wiemken, A (1990). Trehalose in yeast, stressprotectant rather than reserve carbohydrate. Antonie van Leeuwenhoek 58:209-217. 